\title{
En milieu rural, la communication échappe au schéma classique
}

\section{Bernard Dagenais}

\section{(2) OpenEdition}

12 Journals

Édition électronique

URL : http://journals.openedition.org/communicationorganisation/1734

DOI : 10.4000/communicationorganisation. 1734

ISSN : $1775-3546$

Éditeur

Presses universitaires de Bordeaux

\section{Édition imprimée}

Date de publication : 1 novembre 1994

ISSN : 1168-5549

\section{Référence électronique}

Bernard Dagenais, «En milieu rural, la communication échappe au schéma classique »,

Communication et organisation [En ligne], 6 | 1994, mis en ligne le 26 mars 2012, consulté le 04 mai

2019. URL : http://journals.openedition.org/communicationorganisation/1734 ; DOI : 10.4000/ communicationorganisation. 1734

Ce document a été généré automatiquement le 4 mai 2019.

(c) Presses universitaires de Bordeaux 


\section{En milieu rural, la communication échappe au schéma classique}

\section{Bernard Dagenais}

1 De nombreuses études portant sur la place du citoyen dans la démocratie prennent comme point d'ancrage la ville. "C'est le niveau de gouvernement le plus près des citoyens qui traduit le plus clairement les problèmes réels de la population et les solutions qui s'imposent » (Secrétariat des conférences 1978).

2 Mais au fil des ans, la perception de la ville, comme lieu d'accomplissement, se modifie. «D'espace pacifié et policé la ville est devenue la confrontation brute d'egos désocialisés. Elle est perçue au mieux comme espace d'indifférence à la solitude angoissante, au pire comme espace d'insécurité, contre lequel peuvent seuls nous prémunir les douceurs du foyer ou d'hypothétiques armures névrotiques ou chimiques »(Roman 1994, p. 11).

3 Aujourd'hui la ville est décrite comme un lieu d'insécurité, de perdition et de crises constantes. Pour Roman (1994, p.9), la ville est ainsi le lieu où viennent aujourd'hui converger des crises dont « une crise des formes instituées de la communication sociale, de l'échange politique, de l'espace public et ultimement de la représentation politique ». Il rejoint ainsi la vision partagée par plusieurs que la ville est source de dépersonnalisation et d'antagonisme plutôt que le lieu des identités et des dépassements intérieurs. À ce titre, les villages sont devenus des endroits où s'épanouissent les collectivités locales et où les individus retrouvent leur dignité.

4 Le citoyen dans la ville est un individu complexe qui perd toute identité dans le concept d'opinion publique que livrent les sondages. On sait ce qu'il pense, de quoi il a peur et pour qui il veut voter. Mais comme il ne s'administre jamais de sondage dans les petites municipalités, la voix du peuple se fait souvent peu entendre. Les élus reconstruisent le profil de l'opinion publique à partir des échos qui leur parviennent des discussions du bistro ou de l'épicier du coin. Ce qu'on en connaît relève de l'intuition, de l'expression de certaines passions déchainées, ou des intrigues des groupes d'intérêts.

5 Enfin, parler de communication en milieu municipal, c'est étudier les structures ou les stratégies de communication qui privilégient habituellement l'information de service et négligent celle qui est utile pour la prise de décision des citoyens. Ainsi, une population 
sera facilement informée des grandes orientations et décisions qui concernent la vie municipale mais sera incapable de connaître les véritables raisons qui ont orienté certains autres choix politiques. Le citoyen devra avoir recours aux médias pour suivre le comportement des élus municipaux, pour exprimer son mécontentement et faire connaître ses doléances. Et c'est pour eux qu'il organisera des manifestations de protestation.

6 Même si de plus en plus, les municipalités de toute taille ont recours aux communications pour développer leur image, pour faire connaître leurs services, ou pour créer une certaine cohésion dans le milieu qu'elles administrent, même si les médias couvrent les activités municipales comme ils couvrent les autres ordres de gouvernement en privilégiant les activités de législation et de controverse, le citoyen subit plutôt qu'il ne participe à la communication. "La communication publique ne favorise guère les interactions et les échanges : sa fonction est surtout de tenir à distance les sujets citoyens, en les associant par le discours et l'image à la modernisation des administrations » (De la Haye et Miège 1983).

7 Pour contrer cette situation, certains auteurs comme Castagnet (1991, p. 6) proposent un modèle de communication qui permettrait une meilleure circulation de l'information dans la ville. Et Langenieux-Villard (1985, p. 3) n'hésite pas à affirmer que « S'il agit sans communiquer, l'élu perd le pouvoir. S'il communique sans agir, il trompe les citoyens ».

8 Pour Demers (1991), «C'est toutefois autour de la communication municipale que s'articulent les fondements de la démocratie. Premier niveau d'intervention politique, la municipalité représente le lieu où toute décision politique a des répercussions directes sur la qualité de vie des résidents; et où tout mécontentement populaire peut entraîner des changements politiques radicaux ».

«L'étude de la communication municipale débouche nécessairement sur l'analyse de la dimension démocratique de la vie municipale, sur l'utilisation des différentes techniques de communication pour gérer l'espace municipal tout autant que le sentiment d'appartenance et sur le rôle complexe et toujours difficile à évaluer des médias d'information ".

9 Si la communication est l'échange d'information entre deux pôles et non seulement la diffusion d'information d'un pôle à l'autre, on pourrait presque dire qu'en fait, contrairement à ce qu'on lit sur le sujet, il n'existe pas de communication en milieu municipal. Les différents partenaires se livrent plutôt à des monologues qui s'expriment dans les médias. Des études sur les bulletins municipaux confirment qu'ils sont d'abord et avant tout des instruments de valorisation des élus. La recherche de Lavigne (1993 p. 402) confirme «que le bulletin municipal n'est pas une publication très ouverte à la participation, mais plutôt un outil unidirectionnel de relations publiques destiné à légitimer le gouvernement $»$.

Ainsi, «Le rôle de l'information municipale n'en n'est pas pour autant unanimement reconnu. Elle reste l'une des activités communales les plus contestées et l'une des plus difficiles à justifier par le pouvoir en place » (Langenieux-Villard, 1985, p. 4).

10 Or, dans les collectivités rurales où il n'y a ni préposé aux communications, ni structure organisée, ni stratégie manifeste de communication, cette dernière est pourtant omniprésente. Le monde politique se construit autant, sinon davantage, au rythme des rumeurs; le discours se compose selon les amitiés et inimitiés personnelles; la réalité se définit à partir des éloges que propagent les chantres du pouvoir ou des procès d'intention qu'entretiennent quelques mauvaises langues. En fait, il existe une véritable 
communication de face à face. Ces réflexions démontrent que la théorie de la communication en milieu municipal s'est trop souvent satisfaite d'approches globales. Les petites collectivités rurales échappent à la dynamique que créent les grands ensembles urbains. Par ailleurs, elles sécrètent une dynamique originale qui leur est propre.

11 Deux idées de base reviennent dans la littérature sur la communication en milieu municipal. D'une part, la place du citoyen dans la démocratie municipale est déifiée. Toute la littérature nous rappelle que le gouvernement local est celui le plus près du citoyen. Et c'est là seulement que l'un et l'autre peuvent s'exprimer en toute liberté. On parle de proximité et de partenariat, ce qui manque aux ordres plus grands de gouvernement.

D'autre part, on a tendance à montrer comment la communication en milieu municipal peut faciliter l'adhésion des citoyens aux grandes orientations proposées par les élus. Dans la majorité des cas, les études démontrent l'efficacité ou la nécessité de la communication autour des grands débats, des grandes orientations ou même des grandes idéologies politiques. À trop vouloir démontrer que le monde municipal s'est mis à l'heure de la magie de la communication - ou devrait s'y mettre - on oublie que la communication n'est pas une fin mais un moyen de démocratie. On peut utiliser l'information à des fins démagogiques, de propagande, d'images. Or la démocratie véritable repose sur la libre circulation de l'information utile à la prise de décision et non sur la quantité d'information qui circule dans une municipalité.

On s'imagine trop souvent que l'existence d'une stratégie de communication porte en soi des vertus qui vont agir de façon magique sur la réalité.

No étude s'articule autour de l'idée que la communication dans les collectivités rurales échappe à toutes les règles de communication habituelles. Pourtant le discours qui l'entoure l'assimile aux communications classiques des organisations. De ce fait, il existe un fossé entre les faits et gestes des élus municipaux et le discours qu'ils tiennent sur la communication en milieu rural. Ce fossé est entretenu autant par le chercheur que par le praticien des communications municipales qui reproduisent un discours normatif sur ce type de communication. Ainsi, les recherches qui portent sur les techniques de communication essaient d'abord d'en démontrer les bienfaits comme technique au delà du contenu véhiculé.

Pour discuter de ces questions, nous avons choisi le contexte québécois et tout particulièrement l'expérience acquise dans un petit village. La majorité des théories sur la communication en milieu municipal ont tendance à chercher des communs dénominateurs qui englobent l'ensemble du système municipal mais qui ne répondent à aucun des éléments particuliers de ce système. Nous avons voulu décrire un exemple concret qui ne peut être érigé en théorie mais qui traduit bien une réalité particulière. En somme, nous avons voulu savoir s'il y avait adéquation entre la littérature qui porte sur le sujet et notre propre expérience de près de 15 ans (1979-1993) comme maire d'une commune au Québec, Sainte-Pétronille à l'Ile d'Orléans.

16 Nous allons développer notre exposé en décrivant d'abord le contexte de référence. Nous traiterons ensuite de l'originalité de la communication en milieu rural. Et nous conclurons en essayant de comprendre la place du citoyen dans la démocratie municipale. 


\section{Le contexte de référence}

17 Le contexte de référence servira à montrer que lorsqu'on parle de communication en milieu municipal, il faut distinguer les villes dans lesquelles des groupes divergents s'opposent et les villages où les collectivités locales sont plus homogènes et où peuvent régner une tranquillité et une sécurité exemplaires.

\section{Le cadre géographique}

18 Sainte-Pétronille est une des six paroisses-villages de l'Ile d'Orléans. Celle-ci est située à quelque 15 kilomètres du centre-ville de Québec, au milieu du fleuve Saint-Laurent qui fait plus de cinq kilomètres de large à la pointe de l'Ile. Celle-ci est reliée à la terre ferme par un pont.

19 L'histoire a forgé pour chacun des villages de l'ile une personnalité propre. Compte tenu de son site sur la pointe de l'île la plus rapprochée de Québec, Sainte-Pétronille a attiré la grande bourgeoisie anglaise de Québec qui, au siècle dernier, a fait de ce village son lieu de villégiature privilégié. On signale même que le club de golf de Sainte-Pétronille serait le plus vieux en Amérique du Nord.

Dans le dépliant de la Chambre de commerce de l'Ile d'Orléans (1994, p. 2), on définit le village comme suit: «Connue sous l'appellation populaire du «bout de l'Ile», SaintePétronille de Beaulieu fut un endroit de villégiature très populaire au milieu du XIXe siècle. Plusieurs bourgeois de la ville de Québec y firent construire de très belles résidences que l'on peut encore admirer le long du Chemin Royal ».

21 L'Ile d'Orléans, c'est la campagne. Elle est le lieu d'arrêt des migrations saisonnières des oies, outardes, canards, pluviers, hérons. Ses berges subissent des marées quotidiennes de trois à six mètres. C'est le règne de la tranquillité et la fuite de la cohue et des dangers urbains. C'est aussi la campagne à la ville. À vingt minutes des salles d'opéra, de théâtre, de cinéma, du hockey professionnel, d'excellents restaurants et de la vie urbaine, la ville est à portée de la main.

22 L'Ile a une longueur de 34 kilomètres et une largeur de 8 kilomètres. Sa population approximative est de 7000 personnes durant l'hiver et 10000 durant l'été. SaintePétronille, avec ses $4,5 \mathrm{~km} 2$ est le plus petit village. La superficie des autres villages dépasse les $30 \mathrm{~km} 2$. Sa population de 1060 habitants la place au $3^{\mathrm{e}}$ rang de l'ille.

23 L'agriculture est la principale activité de l'Ile. Et le tourisme vient très loin derrière. II s'agit donc d'un site exceptionnel, reconnu pour sa qualité de vie, sa faible densité et son caractère champêtre.

\section{Le cadre de tutelle}

Compte tenu de son caractère exceptionnel, l'Ile a fait l'objet d'une attention bien particulière de la part du gouvernement du Québec. D'abord consacrée site historique en 1970, l'Ile doit répondre à la loi des monuments et sites qui règlemente de façon très précise l'occupation des sols, les aires de protection et de construction. C'est le ministère des Affaires culturelles et des Communications qui gère les modalités de construction ou de démolition de tout bâtiment, jusqu'au moindre petit hangar. Aucune amélioration ne 
peut se faire sur la propriété des habitants sans qu'un permis du ministère de la Culture et des Communications ne soit émis. Et les autorités municipales, encore moins les citoyens, ne peuvent discuter de ces paramètres.

Comme suite à cette "consécration", les fonctionnaires du ministère de la Culture ont recensé à Sainte-Pétronille, une centaine de maisons dites classées, soit près du tiers des maisons du village. L'architecture de celles-ci est défi nie de façon précise et doit être maintenue dans ses moindres détails. Les autorités municipales et les citoyens n'ont rien eu à dire dans cette décision, ni dans son appli cation qui a été prise par des « spécialistes » qui n'ont pas consulté les gens.

Dans une loi cadre, le ministère de l'Agriculture du Québec a décrété que l'Ile d'Orléans était un territoire agricole. A l'exception des coeurs de village où les constructions résidentielles sont permises, $90 \%$ du territoire est désormais dédié de façon absolue à l'agriculture Encore ici, ni les autorités municipales, ni les citoyen n'ont eu leur mot à dire.

Comme sur cette Ile, il n'y a ni égout collecteur, ni aqueduc, tout le territoire est soumis aux directives des fonctionnaires du ministère de l'Environnement qui gère ainsi d'une certaine façon l'occupation des sols. Il s'agit tantôt de lois, tantôt de règlements, ou de directives appelées à changer selon l'évolution de la pensée de ceux qui les émettent. Les élus locaux et la population n'ont rien à dire.

Dans un petit village paisible comme Sainte-Pétronille il n'y a pas de corps policier. Cette tâche est assurée par la Sûreté du Québec, c'est-à-dire par la police de l'État québécois. Cette police applique les lois qui relèvent du code criminel et du code provincial de la route. La Loi de la Police qui pose le cadre de gestion de tout corps policier dans les cités et villes, a oublié qu'une municipalité de 1000 habitants ne se gérait pas comme une ville d'un demi-million d'habitants. Les autorités municipales et les citoyens n'ont aucun mot à dire dans la préparation de ces deux codes. Par ailleurs, cette police qui impose des lois préparées ailleurs, refuse d'appliquer les règlements municipaux et est très peu présente lorsque les citoyens expriment des doléances.

29 La municipalité est gérée par le code municipal qui définit toutes les marges de manœuvre du maire et de ses conseillers. Ce code a un principe : il est interdit d'interdire. Ainsi, une municipalité ne peut décréter qu'elle ne veut pas de terrain de camping sur son territoire. Elle ne peut que choisir les endroits où il y en aura. Ce code municipal qui définit jusque dans ses moindres détails tous les gestes d'une petite municipalité est imposé aux élus municipaux et aux citoyens sans que ceux-ci ne puissent dire quoi que ce soit.

30 Enfin, le gouvernement provincial ne se prive pas, dans les périodes de restriction budgétaire, pour refiler aux municipalités certaines responsabilités sans compensation financière.

31 C'est dans ce carcan - ou cette camisole de force - que va s'exercer la démocratie municipale avec ses grands principes. Si le Conseil municipal a le choix des rues qu'il va paver, il doit suivre des règles strictes pour les appels d'offre, pour le financement des travaux et pour l'octroi des contrats. Et somme, toute même pour le choix des rues, ce sont les citoyens qui décident. L'ordre de priorité de la réfection des rues répond à un savant dosage dont l'état de la chaussée n'est qu'un élément très secondaire. Car, par le biais du financement du projet, avec quelques dizaines de signatures seulement, les 
citoyens ont le pouvoir de s'opposer à toute décision en exigeant la tenue d'un référendum.

Or, lorsqu'on parle de communication et de démocratie municipale, on oublie cette réalité première que dans les petites collectivités, les grands enjeux étant définis ailleurs, la vie municipale s'organise en grande partie autour des rivalités de clans et de familles, ou d'égoïsmes exacerbés.

Péron (1983, p. 297) faisait justement remarquer que «Les relations publiques administratives à caractère communal ne s'exercent bien entendu qu'au sein de l'institution communale qui n'est elle-même qu'un rouage au sein d'un système économique, politique et administratif infiniment plus large ».

Tous ces encadrements limitent de beaucoup la marge de manoeuvre du politique et rendent caduque toute intervention de la population en ces domaines. C'est donc ailleurs que va s'exercer le pouvoir des uns et des autres.

\section{Le cadre social}

Sur le plan social, la moitié des familles de Sainte-Pétronille habitent depuis plusieurs générations sur les mêmes terres. Ce sont les nés natifs. Et l'autre moitié est composée des étrangers venus s'établir à l'Ile depuis quelques dizaines d'années tout au plus. Les premiers savent que l'Ile leur appartient. Ils considèrent que les autres ne font qu'en profiter et nourrissent à leur endroit des sentiments mitigés (Dagenais 1989,1994).

Le pouvoir véritable repose entre les mains des nés natifs. C'est un pouvoir moral. Ils possèdent l'Ile, les commerces, les services, les terres, le savoir faire, la tradition. Ils imposent leurs règles par le jeu très fort des coalitions de famille qui peuvent rapidement faire bloc contre l'individualité des nouveaux arrivants. Mais ils ne possèdent pas le nombre. La démocratie s'inscrit en faux. Les plus forts sont les moins nombreux mais ils ont la capacité de mobiliser rapidement le clan familial selon des intérêts immédiats bien sentis.

La démocratie municipale, c'est de ne pas déplaire aux anciens et de permettre aux nouveaux de garder toute leur place. La marge de manoeuvre se rétrécit encore.

Enfin, il existe deux grands partis politiques au Québec, le parti libéral, parti de (centre) droite et des affaires; le parti québécois, parti de (centre) gauche et des idéologies sociales. Dans l'lle, les deux partis ont des assises bien ancrées. Il faut donc surveiller les faits et gestes qui pourraient indisposer les uns ou les autres.

Il existe aussi des individus qui considèrent que leur village est leur lieu de résidence. Ils recherchent une vie paisible et s'accommodent des inconvénients de la vie en collectivité. Il y en a d'autres pour qui tous les maux de l'existence sont dus à une mauvaise gestion municipale. S'il y a tempête de neige pendant deux jours, les autorités auraient du prévoir et réquisitionner l'équipement et le personnel nécessaires pour que cette tempête n'occasionne aucun inconvénient. S'il fait chaud et que les puits s'assèchent, les autorités municipales devraient mettre un aqueduc.

Mais si on augmente les taxes pour satisfaire une des exigences de cette population, c'est qu'on a été mauvais gestionnaire et qu'on n'aurait pas dû gaspiller les fonds publics par des politiques d'embellissement par exemple. Mais, si on ne met pas de fleurs, c'est qu'on néglige l'image de la municipalité. 
40 La démocratie municipale dans ces circonstances se limite à satisfaire le groupe qui revendique des changements avec le plus de ténacité et non à décider en fonction d'une certaine logique ou d'une certaine rationalité.

41 Péron (1983, p. 287) résumait cette situation en ayant recours à l'euphémisme suivant: «Au plan local, cette compétence [du conseil municipal] est aussi limitée par la liberté des particuliers».

\section{Le cadre administratif}

42 Avec ses quelque 1060 habitants, Sainte-Pétronille n'est pas une exception dans le panorama municipal du Québec. Sur les quelque 1500 municipalités, signalons qu'il y en a quelque 1300 de moins de 5000 habitants et quelque 700 qui comptent une population de plus ou moins 1000 habitants (Ministère des Affaires municipales 1994, p. 41). Mais comme ces municipalités ne représentent que $6 \%$ de la population, elles deviennent quantité négligeable. Et c'est ce qui explique pourquoi les théories sur la vie municipale négligent cette réalité.

43 Il y a six conseillers municipaux et un maire qui sont élus pour être à l'écoute de la population composée de leurs voisins, parents, amis, ennemis et inconnus. Les conseillers n'étant pas affectés à une zone particulière du village, ils représentent l'ensemble du village. Le citoyen qui veut se plaindre en parle à son ami le conseiller qu'il connaît. Ou vient aux séances du conseil municipal, une fois par mois, où il a droit de poser des questions à la fin de la séance. Comme celle-ci démarre à 20 h00 et peut durer jusqu'à $23 \mathrm{~h} 00$, les vaillants qui veulent poser une question doivent rester jusqu'à la fin, et comprendre qu'ils ne recevront qu'une réponse partielle à leur demande vu la complexité du dossier, de l'heure tardive et de la présence des autres citoyens qui veulent prendre la parole.

44 Mais il faut dire que les séances du conseil ont de quoi rebuter n'importe qui. Le vocabulaire employé et la séquence des informations (adoption du procès verbal, les comptes, la correspondance, le contrat de ci et de ça) donnent l'impression qu'il ne s'agit que d'intendance. Les questions des citoyens à la fin de l'assemblée viennent renforcer cette perception.

\section{La communication officielle}

Quelle communication est-il possible de développer à partir de ces paramètres? On se rend compte ici que les différents écrits sur la communication en milieu municipal occultent davantage la réalité que nous venons de décrire au profit d'un discours généreux sur une communication qui permet aux citoyens de participer à la vie démocratique de façon plus intense.

Dans les faits, le citoyen ne participe à la vie démocratique qu'au moment où il vote. En dehors de cette circonstance, une partie des décisions est en effet prise à son insu ou presque, et l'autre partie veut faire taire les revendications (pas toujours fondées) bruyantes. Les avis publics sont rédigés en une langue légale incompréhensible pour la majorité des citoyens et sont exposés souvent dans des lieux peu fréquentés. Et les seules informations qu'il reçoit servent à rendre compte des activités municipales et des actions entreprises par la municipalité (Courcelles, 1983). 

citoyens si bien que la seule véritable communication en milieu municipal, c'est celle que subit le corps politique. Lorsqu'un groupe de pression est insatisfait d'une décision, il n'hésite pas à organiser pétitions, marches de soutien, manifestations pour contrer les projets auxquels il n'adhère pas. Comme le politique est habituellement très prudent sur ce qui peut toucher à son image, alors il cède en utilisant un discours de conciliation, alors qu'il s'agit en fait de capitulation devant le bruit médiatique. Mais il y a là un vrai geste de communication puisqu'il y a échange et non plus seulement diffusion d'information.

\section{La démocratie municipale}

Le discours sur la démocratie municipale décrit une espèce d'Eden où la proximité des partenaires, le pouvoir de réagir directement aux initiatives controversées permet de maintenir un équilibre harmonieux dans un milieu de vie.

Or, lorsque vient le temps de prendre des décisions, les partenaires naturels du citoyen disparaissent. Le gouvernement provincial d'une part ne tient pas compte des opinions du milieu municipal. D'autre part, ce même milieu municipal ne tient compte de ce que ses électeurs pensent que lorsque ceux-ci manifestent leur mécontentement.

50 de la refonte du zonage agricole, le président de l'Union des Municipalités Régionales de Comté du Québec, Roger Nicolet (1989, p. 4) écrivait ceci : «Par contre, la population régionale, les propriétaires concernés, les instances municipales responsables de l'aménagement du territoire ne sont consultées que symboliquement. On fait fi de l'enseignement d'une décennie de démocratie de participation en matière de planification. Tout cela signifie évidemment un retour à une vision centralisatrice de la gestion qui ne manquera pas de provoquer des erreurs importantes dans une démarche capitale pour l'avenir non seulement de l'agriculture québécoise, mais pour l'ensemble de la société régionale. »

On peut dès lors s'interroger sur le rôle que peuvent jouer les communications dans cet univers où la presque totalité des décisions sont prises sans concertation. La question se pose avec d'autant plus d'acuité, que désormais les individus ne sont plus uniquement intégrés à un milieu de vie, ils sont partenaires du monde. Et pendant qu'ils dissertent sur les malheurs qui sévissent dans les villes et pays étrangers, ils oublient de s'investir dans leur propre communauté.

Pour Choay (1994), «L'appartenance à des communautés d'intérêts divers ne se fonde plus ni sur la proximité, ni sur la densité démographique locale. Transports et télécommunications nous impliquent dans des relations toujours plus nombreuses et diverses, membres de collectivités abstraites ou dont les implantations spatiales ne coïncident plus et ne présentent plus de stabilité dans la durée ».

Roman insiste pour expliquer qu'aujourd'hui la télévision est devenue la nouvelle place publique et que la vraie place publique est désertée. «Enfin, il faut remarquer que si la ville a longtemps été symbolisée par la place publique, agora ou forum dans l'antiquité, espace public de communication par l'écrit au temps des Lumières, c'est qu'elle réalisait cette possibilité de confrontation permanente des individus et des opinions qui s'élaborait, par friction mutuellement consentie, une citoyenneté. (...) Nos places sont 
désertées et battues par les vents tandis que nous nous réfugions pour goûter le bienfondé de nos préjugés derrière notre écran de télévision » (Roman, 1994, p. 11).

Dans ces circonstances, le citoyen occupe très peu de place dans les décisions à prendre dans les petites communautés. S'il est vrai que certaines décisions appartiennent en propre à la collectivité et permettent de développer l'originalité de certains villages ruraux, ces décisions sont le fruit d'une négociation étroite entre les élus et les groupes d'intérêt qui seront touchés par ces décisions. La rationalité froide cède trop souvent le pas aux passions du milieu. Dans ce contexte, il faut comprendre que la communication va s'organiser selon des paramètres tout à fait originaux.

\section{L'originalité de la communication en milieu rural}

\& Le champ de la communication municipale constitue un univers à part. A linstar de la communication publique, elle touche à la fois la dimension politique, partisane et administrative. La vie municipale est en effet ce mélange de services offerts à la population, de décisions orientant le développement du milieu de vie dans lequel ont choisi de vivre les résidents d'une ville et de contestation politique issue des antagonismes qui prennent parfois des proportions inquiétantes dans certains milieux » (Demers 1991).

57 Ainsi perçue, la communication en milieu municipal se cantonne dans le rôle de l'émetteur classique. C'est la municipalité qui parle à ses contribuables, résidents, touristes et investisseurs et qui élabore des stratégies de séduction ou de contrôle. Le gouvernement municipal et son administration se comparent alors à une entreprise de service et de ce fait, adoptent des comportements identiques dans la façon de gérer la communication, s'inspirent des mêmes approches et recherchent la meilleure stratégie pour amener la population à partager ses décisions.

58 Ce réflexe est tellement présent que lorsqu'une politique ne réussit pas à séduire la population, on ne s'interroge pas sur le bien-fondé de celle-ci, mais davantage sur la façon dont on n'a pas su la « vendre » au public. En somme, l'État se comporte comme une entreprise qui doit réussir à faire partager ses objectifs et réalisations à sa clientèle.

59 Nous avons voulu démontrer dans la première partie du texte comment la marge de manœuvre d'un maire d'une petite municipalité est limitée. Il doit apprendre à survivre entre les diktats des paliers supérieurs de gouvernement et les caprices de sa population qui dispose de centaines de sujets d'intervention. Le vrai patron, c'est la population.

Compte tenu de la marge de manœuvre limitée d'un maire d'une petite commune, on peut difficilement transposer le modèle classique de la communication des entreprises à ce type d'organisation. Ce modèle (Lougovoy, Huisman 1981, Wilcox, Ault, Agée, 1986) précise qu'une organisation doit développer des liens de sympathie envers sa clientèle et s'assurer d'être à l'écoute de celle-ci de façon à tenir compte de ses attitudes et comportements dans la prise de décision. Or dans les petites collectivités rurales, on 
assiste à un curieux phénomène inverse : les liens de sympathie tissés par les autorités publiques perdent toute signification lorsque des intérêts particuliers des citoyens sont en jeu. Pour conserver la faveur du vote populaire, il faut satisfaire les exigences des citoyens. Or celles-ci ne relèvent jamais d'une analyse rationnelle d'une situation, mais des inconvénients qu'une situation peut apporter à ses habitudes ou privilèges. Les décisions se prennent rarement en fonction de leur valeur intrinsèque.

61 Ainsi donc, contrairement à une entreprise privée qui choisit de tisser des liens de sympathie avec sa clientèle pour permettre son développement et augmenter ses profits, les autorités municipales cèdent aux revendications de ses citoyens pour garder le pouvoir au mépris de la qualité de la gestion. Poussé à l'extrême, ce raisonnement signifie que pour garder le pouvoir, les autorités sont prêtes à sacrifier des mesures qui sont nécessaires à la collectivité.

62 Ce qui veut dire que le rapport que développe une petite commune avec sa clientèle, n'est pas un rapport d'offre de service comme c'est le cas pour les entreprises traditionnelles, qui ont inspiré les modèles de la communication organisationnelle, mais plutôt un rapport de servitude.

63 S'il est vrai que toute entreprise est soumise à la volonté de sa clientèle qui peut refuser d'acheter son produit, celle-ci ne commande pas l'organisation, elle réagit à elle. Alors que dans le cas des petites municipalités rurales, la clientèle commande et le corps municipal s'adapte. S'il est vrai qu'il existe certains petits despotes locaux, ils prennent toujours appui sur les élites locales et leur marge de décision concerne des intérêts bien circonscrits. À la rigueur, la marge la plus grande d'un maire d'une petite municipalité, c'est l'interprétation des lois et règlements pour favoriser ses amis.

En fait, l'organisation, c'est la société civile et la population; le produit c'est la municipalité. Contrairement à la majorité des organisations qui, elles, ont un pouvoir sur leurs employés, ont une influence sur les consommateurs, les municipalités appartiennent aux voteurs et elles sont en position de dépendance. Ceux-ci ne sont pas la clientèle de l'organisation, mais les dirigeants. Les petites municipalités sont véritablement l'objet et l'otage de la population.

65 Au delà d'un certain seuil de densité, le politique reprend ses droits et impose à une collectivité des destinées que celle-ci peut difficilement contrer. Les gouvernements supérieurs échappent en effet à cet encerclement car il n'y a pas la proximité entre les gens, leurs problèmes et ceux qui les résolvent. Aux niveaux gouvernementaux supérieurs, l'État joue le rôle d'une véritable organisation. Les députés sont, sauf exception, des entités presque aussi impersonnelles que les gestionnaires des grandes sociétés civiles.

Dans les petites municipalités, les citoyens revendiquent sans retenue des biens et services. Ils veulent du réel, du concret et de l'immédiat. Le conseiller municipal habite la même rue, la même campagne, fréquente le même marchand que les autres citoyens. Il est quotidiennement confronté à eux. Ceux-ci contrôlent donc la dynamique de la commune. On est loin de la domination de l'État central sur la vie des contribuables.

Vouloir utiliser les modèles de communication des grands ensembles pour comprendre la dynamique de la communication en milieu rural, c'est fausser la lecture de la réalité. Et utiliser un discours de participation, c'est encore maquiller la situation. Voilà donc comment s'articulent les grandeurs et les misères de la communication en milieu rural. Pourquoi donc le discours est-il si loin de la réalité? 


\section{Discours et réalité} res, le responsable des malheurs des uns ou des autres, l'être qui alimente frustrations et fabulations. Il est devenu le nouveau préposé aux plaintes, et le consolateur des cœurs. La CITE est loin de ses préoccupations.

Dans les petites municipalités rurales, pourtant, le maire ne gère pas un espace juridique, il s'occupe davantage d'une grande famille. Il est le confident des uns, le souffre-douleur

Il est l'invité d'honneur de toutes les naissances, de toutes les inaugurations, de toutes manifestations. Il est celui à qui l'on vient demander des dons, des subventions, des participations à diverses activités. Il est le rempart pour protéger ses citoyens contre toute intrusion extérieure même s'il n'a aucun pouvoir pour la contrer. Il est le fer d'attaque pour combattre les desiderata de ses citoyens même s'il sait qu'il s'agit de velléités. À ce titre, la communication en milieu rural prend des allures familiales. 


\section{La collectivité contre le citoyen}

Lorsque vient le temps de prendre certaines décisions, les autorités municipales privilégient parfois le citoyen, parfois la collectivité. C'est pour ses citoyens qu'un village organise de multiples activités : les loisirs, les fêtes, les activités culturelles ou sportives. C'est également pour eux qu'elle fait aménager bibliothèque, garderie, parc, marché, salle communautaire.

Mais il arrive que la collectivité prend tellement le pas sur les citoyens que bientôt le village s'organisera pour elle contre eux. Ainsi, pour créer de l'emploi, il faut des entreprises. Et ces entreprises polluent, dénaturent le paysage, engendrent des inconvénients pour les riverains, créent des zones industrielles et tous les problèmes sociaux qui s'ensuivent. Au nom de la collectivité, on défendra cet apport à l'économie locale, on épousera les arguments de rentabilité des promoteurs pour se plier au site qu'ils auront choisi, en ignorant les inconvénients que pourront subir les citoyens pendant des décennies.

7 Lorsque la municipalité rurale s'occupe du bien-être de ses habitants, elle avance d'un côté de sains principes de gestion destinés au bien-être de la collectivité mais qui ont comme résultat de bénéficier à quelques-uns; ou alors, elle met sur pied des activités destinées à satisfaire des revendications particulières au nom du bien-être des citoyens. Une petite municipalité rurale devient un centre de gestion des demandes, plaintes, revendications ou privilèges qui lui sont acheminés.

8 Désormais, la communication va continuellement osciller entre des discours qui mettent de l'avant soit des normes et des principes, soit des règles de bon sens, destinés dans un cas comme dans l'autre à écarter ou favoriser des revendications individuelles. Le vrai pouvoir du maire réside dans le choix du discours qu'il tient sur la réalité de façon à privilégier certaines avenues. Le discours ainsi fabriqué n'aide pas à la prise de décision, mais sert à habiller celle-ci d'une certaine rationalité.

\section{Bienfaits et désolation de la vie de village}

9 Le village s'oppose à la ville dans l'imaginaire collectif. Pour certains, encore aujourd'hui, la campagne est le lieu de l'ignorance, des préjugés, des intolérances, alors que la ville est un lieu d'enrichissement car c'est là que s'y propagent la culture, les idées nouvelles, les découvertes scientifiques.

30 Or depuis quelques années, ces notions sont inversées. La ville est devenue le lieu des antagonismes, des violences, de la pollution, de la criminalité, des sans-abri, des quartiers pauvres, de la drogue, de la prostitution et des pires intolérances. Et la campagne avec ses valeurs traditionnelles, sa pureté est devenue le refuge par excellence. C'est là que le créateur trouve sa quiétude pour alimenter son inspiration. Et c'est là que se réfugie le citadin pour échapper à la ville.

81 Mais on est aussi en train de réaliser que l'espace rural mythique subit divers assauts. Les fêtes de village sont «récupérées » à outrance par le mercantilisme des organisateurs et des commerçants. On découvre à la campagne les mêmes crimes qu'à la ville.

Désormais, le discours sur le village s'articule, selon les circonstances, autour de visions bucoliques ou catastrophiques selon la thèse à énoncer. 


\section{Le maire : censeur et prédateur}

83 Le vrai déroulement de la vie dans un village échappe aux autorités municipales et habituellement se réalise envers et contre elles. Dans la majorité des cas, les autorités interdisent tout ce qui est novateur. Mais si le courant impose certaines idées ou certaines nouvelles façons de faire, le maire devient prédateur. Ca devient sa chose, il s'y associera de façon ostentatoire. Il s'attribue toute initiative qui réussit à percer le mur d'indifférence de l'administration publique. Encore ici, le discours sera fabriqué en fonction des intérêts des élites locales.

Fourquet et Murard (1976, p. 50) constataient, au début des années 1970 que, "forme vide», la ville se remplit de contenus divers, étrangers les uns aux autres: ainsi "bourrée », la cité parle toutes sortes de langues (...) Notion vide, immatérielle, et cependant bourrée de sens, d'images, de symboles, la cité est dans le même temps chosifiée et personnifiée, à la fois réceptacle et sujet de l'histoire.

Le village est une réalité complexe. À vouloir le déifier par le discours, on fait l'apologie d'une réalité qui n'existe pas. À vouloir le dénigrer pour ses faiblesses, on en oublie les richesses. À vouloir appliquer aux petites municipalités le discours qui entoure les grands ensembles, on parle de deux univers qui, somme toute, ont peu de chose en commun.

\section{La communication dans l'espace local}

La communication en milieu rural s'articule donc autour de paramètres très particuliers. Et la population visée n'en est pas dupe.

« De façon générale, on constate qu'aucune enquête exhaustive n'a été effectuée jusqu’à maintenant au Québec quant à la situation prévalant dans le monde municipal en matière de communication publique. Par ailleurs, dans son rapport intitulé «Réforme fiscale et démocratie municipale ", publié en 1978 (Chapitre 4, p. 29), la Conférence QuébecMunicipalités pointait deux carences majeures en matière d'information des citoyens: l'accessibilité et la vulgarisation. À quelques reprises depuis ce temps, différents sondages (Notamment un sondage SORECOM 1984) révèlent que, des trois paliers de gouvernement, c'est le palier municipal à l'égard duquel la population se dit la moins bien informée, malgré un degré de satisfaction élevé envers les services municipaux " (Ministère des Affaires municipales 1993, p.2). Mais en temps, elle estime que le palier municipal constitue le palier de gouvernement qui lui inspire le plus confiance et celui qui gère mieux ses affaires (Multi Réso 1992, p. 12).

Pourtant, pour organiser la vie sociale, il faut un système de communication. Or cette vie s'anime autour de systèmes de communication particuliers à chaque milieu. De la rencontre personnelle active dans les petites communautés, à l'utilisation des journaux municipaux ou à la retransmission des séances du conseil municipal sur le câble dans des municipalités plus denses, en passant par les banderoles et les panneaux pour annoncer les fêtes populaires et les événements sportifs, diverses techniques de communication sont utilisées par les autorités municipales pour entretenir le dialogue avec les administrés.

«C'est le secrétaire-trésorier qui cumule, en plus de ses multiples autres tâches, le rôle de responsable de l'information dans un peu plus de $80 \%$ des municipalités » de moins de 5000 habitants. «Pour la plupart d'entre elles, la problématique des 
ressources humaines et professionnelles, financières et matérielles constitue une contrainte lorsque vient le temps d'envisager la communication municipale... » (Ministère des Affaires municipales 1993, p. 2).

89 À côté de ces différents discours sur la réalité municipale, il se fait beaucoup de communication de service dans les municipalités, même les petites. Dans plusieurs municipalités, le bulletin municipal occupe un rôle central d'information. Mais diverses études (Loquay 1987, Fourdin 1988, Dauvin 1990, Le Bart et Dauvin, 1991) démontrent très bien que l'objectif de ces bulletins est de favoriser la réélection des élus locaux et qu'ils sont avant tout des supports promotionnels de l'action municipale.

Dans un village où la rumeur enfle, déforme, trahit, exagère tout, le rôle du maire face aux citoyens, ce n'est pas seulement de dire, mais aussi de taire.

«C'est par l'intermédiaire des médias d'information que s'établit l'équilibre des forces. De multiples études ont démontré comment le pouvoir municipal pouvait exercer des attraits, pour ne pas parler des fréquentations privilégiées, entre certains journalistes et certaines personnalités politiques. À l'inverse, on a assisté à des antagonismes presque malsains entre certains médias et les élus » (Demers 1991).

91 Les médias locaux et régionaux servent enfin de relais aux stratégies des uns et des autres. La vie municipale s'organise parfois par et pour les médias.

En milieu rural, s'il existe un responsable des communications, ce n'est jamais sa tâche principale. S'il existe un bulletin d'information, il traduit davantage les préoccupations du maire que des citoyens. S'il y a un média d'information, il est davantage complaisant que critique à l'égard des élus municipaux (Paletz et al. 1971).

93 Le citoyen peut-il être bien renseigné et participer de façon dynamique à la démocratie face à un tel circuit de communication? La réponse s'impose: en milieu rural, la démocratie se structure selon des paramètres qui n'incluent pas nécessairement la connaissance fine des enjeux sociaux.

\section{L'acteur et l'observateur}

«Entre les théories générales prétendant tout expliquer à partir du paradigme qu'elles ont choisi » (Miège 1989, p 13) et le discours d'expertise, il nous semble possible d'effectuer «un va-et-vient entre l'observation et la théorie » (Bourre 1990 p. 329), à condition de ne jamais oublier la position que l'on occupe et le lieu d'où l'on s'exprime: le chercheur n'a pas vocation à se muer en praticien et réciproquement » (Fourdin 1993, p. 143).

Même si une expérience individuelle ne peut s'édifier en théorie, la connaissance pratique d'une réalité permet parfois au chercheur de prendre conscience de certaines facettes qui demeurent occultées soit par intérêt des acteurs, soit par ignorance.

Entre les règles du jeu qui doivent présider ou expliquer les actions de la relation citoyenespace municipal et le vécu de ces relations surgit une réalité peu prosaïque pour les chercheurs et peu ennoblissant pour les acteurs. De ce fait, les uns et les autres vont construire un univers symbolique autour des données dont ils disposent.

Cet univers symbolique qui tient du discours va acquérir une vie autonome. "On sait depuis Frege que les mots peuvent avoir un sens sans référer à rien. C'est dire que la rigueur formelle peut masquer le décollage sémantique. Toutes les théologies religieuses et toutes les théodicées politiques ont tiré parti du fait que les capacités génératives de la 
langue peuvent excéder les limites de l'intuition ou de la vérification empirique pour produire des discours formellement corrects mais sémantiquement vides » (Bourdieu, 1982, p. 20).

Nous sommes donc confrontés aux grandeurs et misères de la communication en milieu rural qui amènent le citoyen à pratiquer une forme de démocratie de proximité où ce n'est plus seulement la raison et les convictions qui priment mais plutôt une foule de paramètres autant psycho-sociaux qu'économiques qui entrent en ligne de compte.

C'est aussi ce qui explique la richesse et la complexité de la vie rurale. Elle s'anime autour de réalités qu'évacue trop facilement la littérature scientifique.

La communication dans les collectivités rurales répond donc à des enjeux qui dépassent les techniques ou les stratégies utilisées dans l'une ou l'autre collectivité. C'est toute l'organisation sociale qui revit à travers elle.

\section{BIBLIOGRAPHIE}

BOURDIEU Pierre, Ce que parler veut dire : l'économie des échanges linguistiques, Fayard, 1982, $244 \mathrm{p}$.

BOURRE Robert, « La recherche sur contrat transforme-t-elle l'universitaire en chercheur à gages ? " Congrès de la SFSIC, «L'avenir de la recherche en information-communication », Aixen-Provence, 24-26 mai, 1990, p. 325-331.

CASTAGNET, Michel, « Les règles du jeu d'une communication locale », Territoires-Correspondance municipale, mai, 1991, pp. 6-9.

CHAMBRE DE COMMERCE DE L'ILE D'ORLEANS,, L'Isle d'Orléans : l'Ile ensorcelée, 1994, 50 p.

CHOAY Choay, «Le règne de l'urbain et la mort de la ville », in La Ville, art et architecture en Europe, 1870-1993, Paris Centre Georges-Pompidou, 1994.

COURCELLES Lise, « La presse municipale : une analyse », Municipalité, février, 1983, pp. 7-9.

DAGENAIS Bernard, «Cable Television's Socio cultural Impact Difficult to Assess ». Canadian Journal of Communication. Printemps, 1989, vol. $14 \mathrm{n}^{\circ} 2$ 2. pp. 81-88.

DAGENAIS Bernard," «L'écoute de la télévision et l'arrivée du câble à l'Ile d'Orléans », in Jean Paul Baillargeon et al, Le téléspectateur : glouton ou gourmet, Institut québécois de recherche sur la culture, Québec, 1994, pp. 189 à 210.

DAUVIN Pascal, « Le bulletin municipal de Rennes, souci du lecteur ou de l'électeur ? » Mars, $\mathrm{n}^{\circ}$ 25, décembre, 1990, pp. 65-79.

DE LA HAYE Yves et Bernard Miège, "Ce que cachent les discours sur la communication ", Armand Mattelart et Yves Stourdze, Technologie, culture et communication, Paris, La documentation française, 1983.

DEMERS François, « La communication municipale, un nouveau champ de recherche ", Triangle, Bulletin de l'Association des communicateurs municipaux du Québec, mars 1991, volume VI, ${ }^{\circ}$ 1,1991 . 
FOURDIN Monique, «Le magazine : Ville de Paris », Territoires-Correspondance municipale, novembre, 1988 , pp. 41-43.

FOURDIN Monique, «Communication urbaine : l'apport des représentations sociales », Sciences de la société, nº 30, octobre, 1993, pp. 141-156:

FOURQUET François et Lion Murard, Les équipement du pouvoir, U.G.E. 10 \18, Paris. Cité par Robert Boure, « Deux ou trois choses que l'on sait d'elle... », (p. 5) in Sciences de la Société, nº 30, octobre 1993, p 5-7)

LANGENIEUX-VILLARD, L'information municipale, PUF, Paris, Que sais-je n² 2245, 1985, 128 p.

LAVIGNE Alain, L'information des gouvernements municipaux québécois en contexte électoral : système d'information et bulletin municipal de trois villes de la Communauté urbaine de Québec, Thèse de doctorat, Département de science politique, Faculté des sciences sociales, Université Laval, 1993.

LE BART Christian et Pascal Dauvin, « Presse municipale, presse locale, un discours préconstruit », Médiapouvoirs, no 21, janvier-février-mars, 1991, pp. 53-60

LOQUAY Philippe 1987, Le bulletin officiel local ? Analyse fonctionnelle comparative des bulletins d'information des collectivités locales et territoriales du département de la Gironde, Université de Bordeaux 3 , Thèse de $3 e$ cycle en science de l'information et de la communication.

LOUGOVOY C. et D. Huisman, Traité de relations publiques, PUF, Paris, 1981, 646 p.

MIEGE B., La société conquise par la communication, Grenoble, PUG, 1989.

MINISTERE DES AFFAIRES MUNICIPALES, «Projet d'élaboration d'un guide de la communication municipale », préparé par la Direction des communications et daté du 22 février, 1993,.

MINISTERE DES AFFAIRES MUNICIPALES, Répertoire des municipalités du Québec, 1994, Les Publications du Québec, 1994.

MULTI RESO « Sondage Multi Réso : municipalités $59 \%$, Provincial $40 \%$, Fédéral $31 \%$ ». Urba, novembre, 1992, pp 12-15.

NICOLET Roger, « Zonage agricole : Au-delà du dérapage et de l'enlisement », Union des Municipalités Régionales de Comté du Québec, 3 juillet 1989, 7 pages.

PALETZ D, REICHERT P, MCINTIRE B. « How the media support local governement authority », Public Opinion Quarterly, Printemps 1971.

PERON Daniel, Relations publiques et information dans les communes, Sirey, Paris,1983, 324 p.

ROMAN Joël, « La ville : chronique d'une mort annoncée ?», Esprit, juin 1994, pp. 5-14.

SECRETARIAT DES CONFERENCES SOCIO-ECONOMIQUES, La revitalisation du pouvoir municipal. Rapport de la conférence Québec-municipalités, Québec, 1978.

WILCOX D, P. AULT, W. AGEE, Public relations: strategies and tactics, Harper \& Row, New York, $1986,646 \mathrm{p}$.

\section{RÉSUMÉS}

Vouloir étudier la place du citoyen dans la démocratie municipale impose un triple questionnement sur les concepts de ville, de citoyen et de communication. L'auteur veut démontrer que les théories et réflexions sur la communication en milieu municipal traduisent 
mal la réalité vécue dans les collectivités rurales et souvent méconnaissent totalement la dynamique particulière de la communication au sein de ces mêmes collectivités.

To study the role of the citizen in municipal democracy, one must first question the concepts of city, citizen and communication. This article shows that the theory and reflexions on the subject of communication and municipality give a wrong view of the reality of communication in rural villages and most often ignore the special dynamic of interrelation between politics and people in these small surroundings.

\section{AUTEUR}

\section{BERNARD DAGENAIS}

Bernard Dagenais est professeur agrégé au Département d'information et de communication de l'Université Laval. Il s'est particulièrement intéressé au rôle des médias dans la société. Il a publié Le communiqué ou l'art de faire parler de soi (Montréal, VLB 1990), La crise d'octobre au Québec et les médias, (Montréal, VLB 1990) et avec Marc Raboy (ed) Media, crisis and democracy : Mass communication and the disruption of social order, (Londres, Sage, 1992). Bernard Dagenais a été maire du village de Sainte-Pétronille, au Québec, de 1979 à 1993. 